\title{
YTHDF2, a protein repressed by miR-145, regulates proliferation, apoptosis, and migration in ovarian cancer cells
}

\author{
Jie $\mathrm{Li}^{1 *} \mathbb{D}$, Lei $\mathrm{Wu}^{2}$, Meili Pei ${ }^{2}$ and Yun Zhang ${ }^{1}$
}

\begin{abstract}
RNA methylation can reverse the methylation modification at the RNA level, which is an extremely important epigenetic modification. The function and mechanism of YTHDF2, as a reader of m6A modification, in epithelial ovarian cancer (EOC) have not been elucidated so far. This study aimed to investigate how YTHDF2 and miR-145 modulated EOC progression through m6A modification. It demonstrated that YTHDF2 was significantly upregulated in EOC tissues compared with normal ovarian tissues. Further functional studies confirmed that YTHDF2 significantly promoted the proliferation and migration of EOC cell lines and reduced the global 6-methyladenine (m6A) mRNA levels. Next, the expression levels of miR-145 and YTHDF2 were found to be inversely correlated in ovarian cancer tissues and cells, and YTHDF2 was the direct target gene of miR-145. A crucial crosstalk occurred between miR-145 and YTHDF2 via a doublenegative feedback loop. The overexpression of YTHDF2 rescued miR-145-induced reduction of the proliferation and migration of EOC cells. Hence, YTHDF2 and miR-145, as two crucial m6A regulators, were involved in the progression of EOC by indirectly modulating m6A levels. The findings of this study on YTHDF2 and miR-145 might provide new insights into carcinogenesis and new potential therapeutic targets for EOC.
\end{abstract}

Keywords: YTHDF2, m6A, miR-145, Ovarian cancer

\section{Introduction}

Ovarian cancer is a common malignant tumor of the female reproductive system, and its mortality rate ranks first among gynecological tumors [1]. It has many histopathological types, about $90 \%$ of which belong to epithelial ovarian cancer (EOC). Patients with EOC have no obvious symptoms and signs in the early stage and lack effective means of early screening. Consequently, most of them reach the late stage before a clear diagnosis. The main treatment of EOC is surgery, supplemented by intravenous chemotherapy or intraperitoneal perfusion chemotherapy or combination therapy. However, the treatment has not achieved satisfactory results; the 5-

\footnotetext{
* Correspondence: lijiexj64@126.com

'Department of Pathology, the First Affiliated Hospital of Xi'an Jiaotong University, 277 West Yanta Road, Xi'an 710061, Shaanxi, China

Full list of author information is available at the end of the article
}

year survival rate of patients with EOC is still around $40 \%$ [2]. Besides the difficulty in early diagnosis, the poor prognosis of patients is also attributed to tumor recurrence and metastasis [3]. Therefore, early sensitive markers of EOC should be discovered, key molecules related to EOC recurrence and metastasis should be identified, and a targeted and multi-functional gene therapy should be developed on an urgent basis.

RNA methylation can reverse the methylation modification at the RNA level, which is an extremely important epigenetic modification [4]. Further, 6-methyladenine (m6A) is the most abundant methylation modification of mRNA in eukaryotic cells, involving the participation of three types of molecules: methyltransferase complex (METTL3, METTL14, and WTAP), named as "Writers"; demethylase (FTO and ALKBH5), named as "Erasers"; and m6A-modified binding protein (YTHDF1/2/3), 
named as "readers", which can dynamically and reversibly regulate the m6A level $[5,6]$. In 2017, Professor Chuan's research team found that $\mathrm{m} 6 \mathrm{~A}$ was involved in the regulation of self-renewal and differentiation of glioblastoma stem cells (GSCs). In vitro and in vivo experiments showed that the knockout of FOXM1-AS and ALKBH5 affected the tumorigenicity of GSCs related to FOXM1, revealing the importance of demethylase ALKBH5 and m6A in glioblastoma [7]. Shuhan et al. revealed the significance of METTL14 in inhibiting the metastasis of HCC [8]. Jaffrey et al. found that METLL3 regulated the myeloid differentiation of normal hematopoietic stem cells and leukemic cells, thus providing more basis for METTL3 as a potential target for myeloid leukemia treatment [9].

Readers are responsible for "reading" the information of RNA methylation modification and participating in the process of downstream RNA translation and degradation. "Reading" has two modes. One is direct reading, which refers to the selective binding with the m6A site of RNA. The earliest readers belong to the YTH domain family of proteins, including YTHDF and YTHDC subtypes, such as YTHDC1, YTHDC2, YTHDF1, YTHDF2, and YTHDF3 [10]. YTHDF2 can accelerate the decay of m6A methylated mRNA [11, 12]. YTHDF2 was found to regulate m6A levels in HCC [13]. However, the expression and mechanism of YTHDF2 in most tumors, especially in ovarian cancer, have not been elucidated so far.

MicroRNAs (miRNAs) are a kind of endogenous noncoding microRNAs with a length of about $22 \mathrm{nu}$ cleotides, which widely exist in eukaryotes. A correlation was observed between the methylation modification of RNA m6A and miRNA. On the one hand, the miRNA-targeting site showed that m6A was enriched, and miRNA could positively regulate the activity of METTL3; on the other hand, the miRNA synthesis depended on m6A methylation modification $[14,15]$. The expression of miR-145 was significantly lower in breast cancer, cervical cancer, glioma, colon cancer, esophageal cancer, and nonsmall cell lung cancer [16]. MiR-145 has different regulatory genes in different tumors; the molecular mechanisms of tumor suppressors are also different. Previous studies confirmed that miR-145 could regulate different biological functions of ovarian cancer by targeting different target genes [17-20]. However, the expression pattern and the m6A-regulated role of miR-145 in ovarian cancer were still unclear.

The present study found that YTHDF2 and miR-145 formed a negative feedback pathway to regulate ovarian cancer progression through m6A modification. The results provided a theoretical basis for the application of YTHDF2 and miR-145 in the diagnosis and treatment of ovarian cancer.

\section{Materails and methods}

\section{Human tissue specimens and cell culture}

SKOV3 was obtained from the Shanghai Cell Bank of Chinese Academy of Sciences (Shanghai, China), 3AO was from the Shandong Academy of Medical Sciences (Jinan, China). Cells were maintained in RPMI 1640 medium (Gibco-BRL, Gaithersburg, MD, USA) supplemented with $10 \%(\mathrm{v} / \mathrm{v})$ fetal bovine serum at $37^{\circ} \mathrm{C}$ under a humidified $5 \% \mathrm{CO}_{2}$ atmosphere. Human ovarian cancer tissue samples and normal ovarian tissue samples were collected from patients at The First Affiliated Hospital of Xi'an Jiaotong University, PR China. This study was approved by the Ethics Committee of The First Affiliated Hospital of Xi'an Jiaotong University, China.

\section{Plasmid transfection}

The human YTHDF2 expression vector pcDNA3-flagYTHDF2 were obtained from Addgene (Boston, MA, USA). Cells were seeded into 6-well plates until 70-90\% confluency and transiently transfected with pcDNA3flag-YTHDF2 or empty vector using the X-treme GENE HP DNA Transfection Reagent (Roche, Indianapolis, IN, USA) following the manufacturer's protocol.

\section{siRNA and transient transfection}

Human YTHDF2 siRNA were purchased from GenePharma (Shanghai, China). YTHDF2 siRNA was transiently transfected $100 \mathrm{nM}$ per well using the X-treme GENE siRNA Transfection Reagent (Roche, Indianapolis, IN, USA) following the manufacturer's protocol. RNA was extracted $48 \mathrm{~h}$ later and protein was extracted $72 \mathrm{~h}$ later for subsequent experiments.

\section{miR transient transfection}

miR-145 mimic and negative control were purchased from Ribo-Bio Co. Ltd. (Guangzhou, China). SKOV3 and $3 \mathrm{AO}$ cells were transiently transfected with $60 \mathrm{nM}$ miR-145 mimic or negative control using the X-treme GENE siRNA Transfection Reagent (Roche, Indianapolis, IN, USA) following the manufacturer's protocol.

\section{Quantitative real-time PCR (qRT-PCR)}

Total RNA was extracted from cells using TRIzol reagent (Invitrogen, Carlsbad, CA, USA) according to the manufacturer's instructions. For mRNA detection, firststrand cDNA was synthesized using a RevertAid first strand cDNA synthesis Kit (Thermo Fisher Scientific Inc., Waltham, MA, USA). Quantitative real-time PCR was performed using a SYBR Premix Ex $\mathrm{Taq}^{\mathrm{Tm}}$ II kit (Takara, Dalian, China) on a CFX96 real-time PCR system (Bio- Rad, Hercules, CA, USA). 


\section{Western blot}

Total proteins were extracted by RIPA lysis buffer (Roche, Indianapolis, IN, USA) and $1 \mathrm{mM}$ PMSF on ice, proteins were separated by SDS-PAGE and then transmembrane. 5\% skimmed milk was sealed at room temperature for $2 \mathrm{~h}$, and then incubated overnight at $4{ }^{\circ} \mathrm{C}$ with rabbit anti-human YTHDF2(1:1000, Cell Signaling Technology, Danvers, MA, USA). TBST membrane was washed for $8 \mathrm{~min}$ and 5 times, and the corresponding second antibody (1:2000) was added, incubated for $2 \mathrm{~h}$, and TBST membrane was washed for 8 min and 5 times.

\section{Luciferase reporter assay}

Cells were co-transfected with pRL-TK vector $(20 \mathrm{ng})$, wild-type (WT-3' UTR) or mutant (MUT-3' UTR) reporter vectors $(180 \mathrm{ng})$, along with miR-145 mimic or negative control at a final concentration of $20 \mathrm{nM}$ using the X-treme GENE siRNA Transfection Reagent. $24 \mathrm{~h}$ after transfection, the relative firefly luciferase activity (normalized to Renilla luciferase activity) was measured using a dual-luciferase reporter gene assay system (Promega, Madison, WI, USA), and results were depicted as the percentage change over the respective control.

\section{RNA m6A quantitative experiment}

In this experiment, the total RNA content of m6A was determined by using the m6A RNA metrology Quantification Kit (ab185912, Abcam) of Abcam company. We measured m6A level following the manufacturer's protocol. The absorbance of the measuring plate at $450 \mathrm{~nm}$ was measured by the enzyme scale instrument, and the RNA m6A content of each sample was calculated according to the standard curve. The formula is $\mathrm{m} 6 \mathrm{~A} \%=$ $[($ sample OD-NC OD)/S] / [(PC OD-NC OD $) / \mathrm{P}] \times 100 \%$, where $S$ is the ng amount of sample RNA and $P$ is the ng amount of positive control RNA.

\section{Cell viability assay}

Cells in logarithmic growth phase were inoculated into 96-well plates with 5000 holes per hole, $100 \mu \mathrm{L}$ of culture medium was added into each hole and incubated overnight in a $37^{\circ} \mathrm{C}, 5 \% \mathrm{CO}_{2}$ incubator, then add berberine for $48 \mathrm{~h} .10 \mu \mathrm{L}$ CCK8(7Sea, Shanghai, China) was added to each pore and incubated at $37^{\circ} \mathrm{C}$ for $4 \mathrm{~h}$. The absorbance value of each pore OD 450 was determined by enzyme labeling (PerkinElmer, Waltham, MA, USA).

\section{Transwell assay}

Cells were trypsinized and counted. A total of $1 \times 10^{5}$ cells in $100 \mu \mathrm{l}$ serum-free medium were added into millicells (Millipore Co., Bedford, MA, USA). $500 \mu$ l of 1640 medium containing 20\% newborn bovine serum was added to the bottom chambers as the chemotactic factor.
After incubation for 24 at $37^{\circ} \mathrm{C}$. Migratory cells were counted and averaged from images of five random fields (original magnification $\times 200$ ) captured using an inverted light microscope. Each cell count was performed by three researchers.

\section{Cell apoptosis assay}

Cell apoptosis analysis was performed using an Annexin V-FITC/propidium iodide (PI) Apoptosis Detection kit (KeyGEN Biotech, Nanjing, China). Normal culture cells were selected in the logarithmic growth period, and the growth state was good for the experiment. After culture for $24 \mathrm{~h}$, the supernatant was introduced into EP tube, and cells were digested with trypsin without EDTA, then cell suspension was made and transferred to new EP tube. After centrifuging for $10 \mathrm{~min}$ at $1000 \mathrm{rpm}$ and $4{ }^{\circ} \mathrm{C}$, discard the supernatant; add $1 \mathrm{ml}$ of precooled PBS, gently blow to suspend the cells for $1000 \mathrm{rpm}$, centrifuging for $10 \mathrm{~min}$ at $4{ }^{\circ} \mathrm{C}$, discard the supernatant; repeat step 3 and step 4 twice; re suspend the cells in $400 \mu \mathrm{l} \times$ binding buffer; add $5 \mu \mathrm{l}$ annexin V-FITC to each sample to be tested, and add PI after mixing $5 \mu \mathrm{l}$, mix well, react at room temperature for $15 \mathrm{~min}$, pay attention to avoid light, and try to get on the machine within $1 \mathrm{~h}$. The results were analyzed using the Cell-Quest ${ }^{\mathrm{tm}}$ Pro software (BD Biosciences, Bedford, MA, USA).

\section{Statistical analysis}

Data were presented as the means \pm SE and were analyzed using SPSS 22.0 software (Chicago, IL, USA). Statistical differences were tested by Chi-square test, two-tailed ttest, one-way ANOVA test or Fisher's Exact test. Differences were considered significant at $P<0.05\left(^{*}\right)$ or highly significant at $P<0.001{ }^{(* *)}$.

\section{Results}

Expression of YTHDF2 in ovarian cancer tissues

An increasing number of studies have shown that m6A modification is vital in the occurrence and development of complex human diseases, especially cancer. However, its specific expression patterns in EOC are still unclear. The present study reported the mRNA expression of YTHDF2 in ovarian cancer tissues and normal ovarian tissues. The expression of YTHDF2 was higher in ovarian cancer tissues than in normal ovarian tissues (Fig. 1a). The clinicopathological correlation analysis of the DNMT3A level in ovarian cancer showed that the later the clinical stage, the higher the pathological grade, the higher the expression of YTHDF2, and the higher the expression of YTHDF2 in patients with metastasis (Fig. 1b-d). The results indicated that YTHDF2 promoted ovarian cancer progression. 

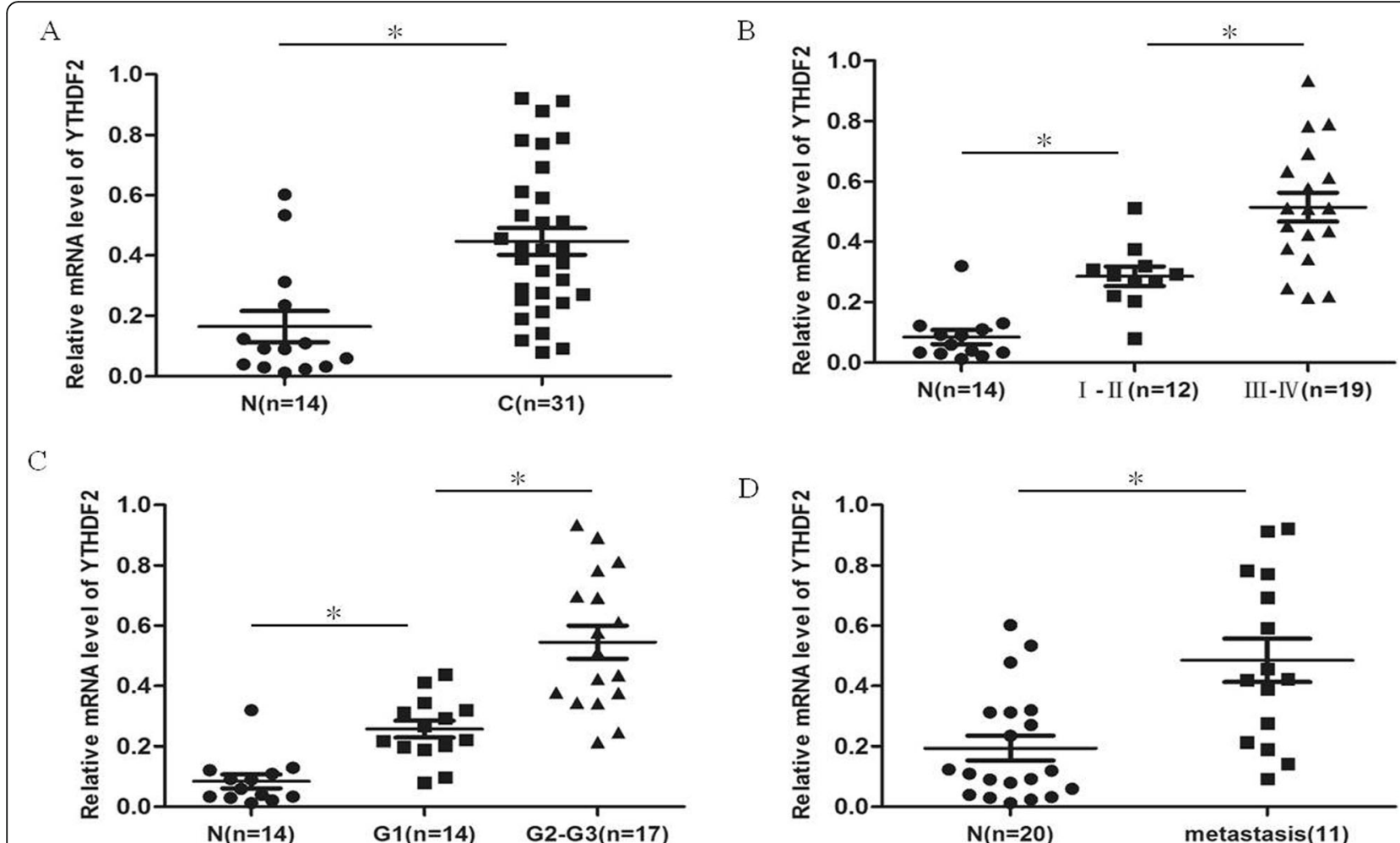

Fig. 1 The expression of YTHDF2 in ovarian cancer tissues. a Relative expression of YTHDF2 in ovarian cancer tissues and normal ovarian tissues. b The relationship between the expression level of YTHDF2 and clinical stage. $\mathbf{c}$ The relationship between the expression level of YTHDF2 and pathological grade. $\mathbf{d}$ The relationship between the expression level of YTHDF2 and metastasis. All experiments were carried out in triplicate and the results were presented as means $\pm \mathrm{SE}$. ${ }^{*} P<0.05$, ${ }^{*} P<0.001$, t-test. $\mathrm{N}$ normal ovarian tissue, $\mathrm{C}$ ovarian cancer tissues

YTHDF2 significantly downregulated global mRNA m6A expression to promote EOC cell proliferation and migration

An RNA m6A quantitative experiment was performed to investigate global mRNA m6A expresssion. After the downregulation of YTHDF2 (Fig. 2a), the global mRNA m6A expression was upregulated (Fig. 2b). The CCK8 test results showed that the proliferation of ovarian cancer cells decreased after knocking down YTHDF2 (Fig. 2c). Accordingly, cell apoptosis increased (Fig. 2d) and migration decreased (Fig. 2e). Next, after the overexpression of YTHDF2 (Fig. 2f), the global mRNA m6A level decreased (Fig. 2g), the proliferation of ovarian cancer cells increased (Fig. 2h), cell apoptosis decreased (Fig. 2i), and migration increased (Fig. 2j). In conclusion, YTHDF2, as an important m6A reader, significantly promoted proliferation and migration by decreasing the global mRNA m6A levels in ovarian cancer cells, suggesting the involvement of $\mathrm{m} 6 \mathrm{~A}$ modification and the reader protein YTHDF2 in the carcinogenesis of EOC.

YTHDF2 was the direct target gene of miR-145

A previous study demonstrated low expression of miR145 in ovarian cancer. The present study further analyzed the mRNA expression levels of miR-145 and YTHDF2 in ovarian cancer tissues. The results showed that the expression levels of miR-145 and YTHDF2 had an inverse correlation in ovarian cancer (Fig. 3a). The YTHDF2 level was higher in the miR-145 low-expression cell line than in the miR-145 high-expression cell line (Fig. 3b). After the overexpression of miR-145 (Fig. 3c), the mRNA and protein expression levels of YTHDF2 decreased (Fig. 3d). The expression level of miR-145 also decreased (Fig. 3e) after the overexpression of YTHDF2 (Fig. 2f), suggesting a crucial crosstalk between miR-145 and YTHDF2 via a double-negative feedback loop. TargetScan (http:// www. targetscan.org/, http://www.mirdb.org/) predicted that YTHDF2 was a target gene of miR-145. The luciferase reporter assay revealed that miR-145 targeted YTHDF2 directly (Fig. 3f). In a word, YTHDF2 was the direct target gene of miR-145.

\section{Overexpression of YTHDF2 rescued miR-145-induced} reduction of the proliferation and migration of EOC cells The overexpression of miR-145 inhibited the proliferation, migration, and apoptosis of ovarian cancer cells, which was attenuated by the overexpression of YTHDF2 (Fig. $4 \mathrm{a}-\mathrm{c}$ ). Similarly, the effect of overexpression of 


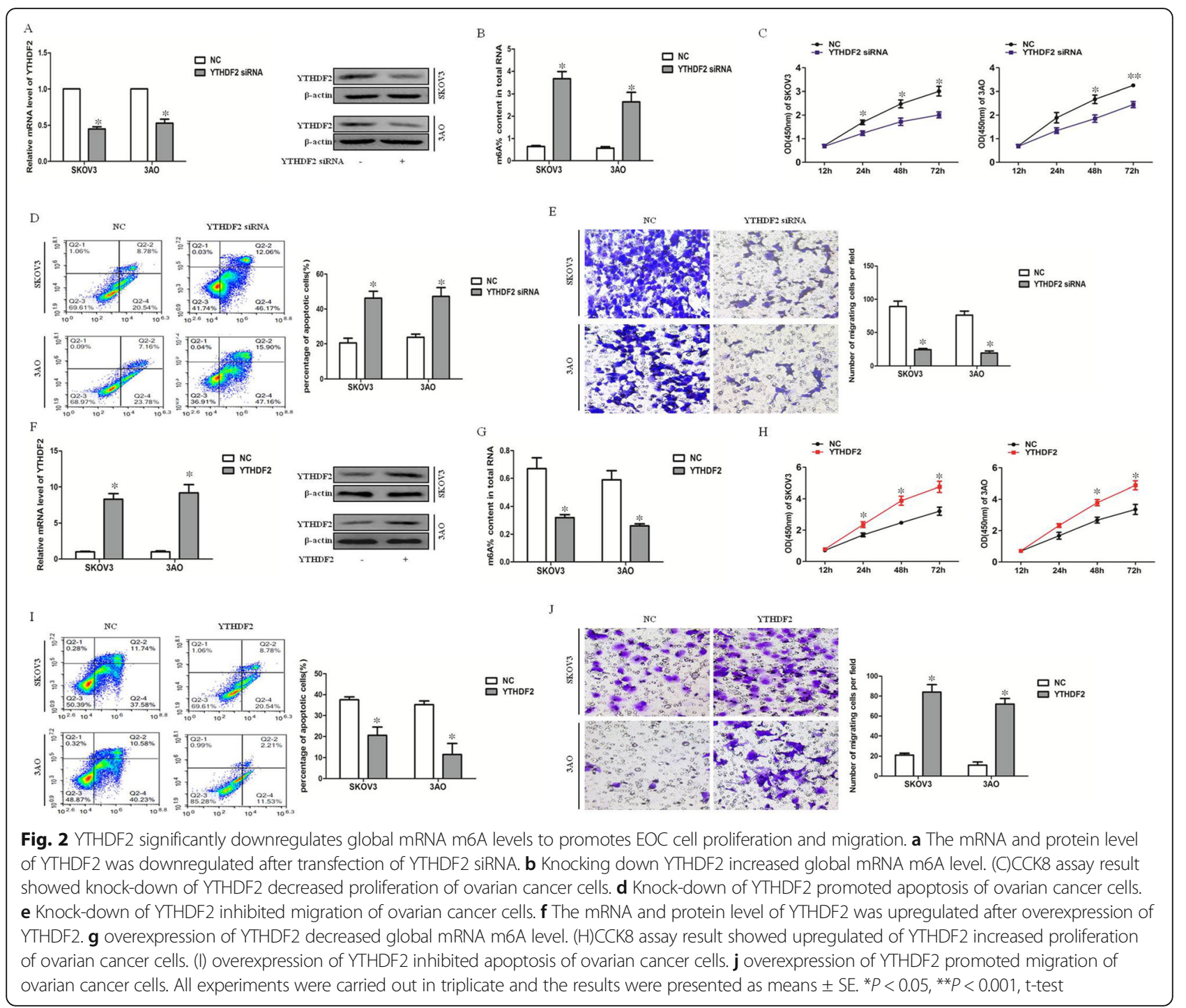

miR-145 on global mRNA m6A levels was offset by the overexpression of YTHDF2 (Fig. 4d). The quantitative real-time polymerase chain reaction and Western blot analysis results showed that, after the overexpression of miR-145, the expression of YTHDF2 decreased, which was reversed by the overexpression of YTHDF2 (Fig. 4e and $\mathrm{f}$ ). To conclude, the overexpression of YTHDF2 rescued the miR-145-induced reduction of proliferation and migration in EOC.

\section{Discussion}

Ovarian cancer is a common malignant tumor of the female reproductive system, and its mortality rate ranks the first in gynecological tumors [1]. Besides the difficulty in early diagnosis, the poor prognosis of patients is also attributed to tumor recurrence and metastasis [2]. However, the specific mechanisms of EOC have not been fully elucidated. Recent studies have focused on the relationship between the methylation of m6A mRNA and tumor development. Increasing evidence shows that the methylation of m6A mRNA is closely related to the occurrence and development of tumors. The expression level of m6A-related protein is an important regulatory factor that directly determines the pathological process of tumor development [6]. However, the m6A modification in the regulation of EOC is still poorly understood. This study investigated how YTHDF2 and miR-145 modulated EOC progression through m6A modification.

Methylation of $\mathrm{m} 6 \mathrm{~A}$ is a dynamic and reversible process, involving writers, erasers, and readers [21]. YTHDF2, as a reader of m6A modification, belongs to the YTH domain family. It is closely related to the malignancy of $\mathrm{HCC}$ and can be adjusted by recognizing the m6A site. The downregulated miR-145 in patients with $\mathrm{HCC}$ can directly target the 3 '-untranslated region of YTHDF2 mRNA to inhibit the expression of YTHDF2, 


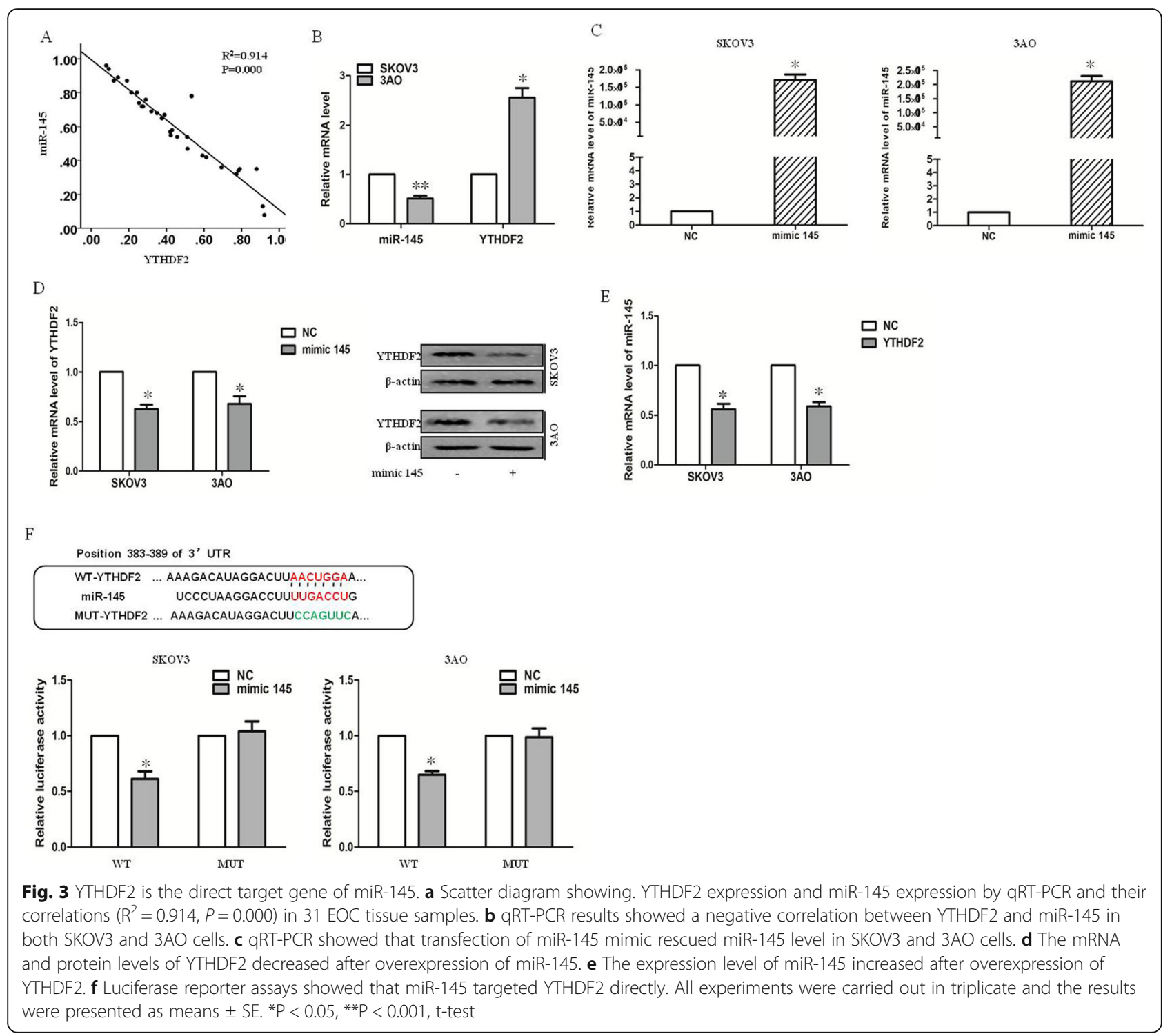

which may be a new target for the treatment of HCC [13]. YTHDF2 is closely related to the malignant degree of HCC and can regulate mRNA degradation by recognizing the $\mathrm{m} 6 \mathrm{~A}$ site, leading to the enhancement of HCC cell proliferation [22-24]. However, the role of YTHDF2 in ovarian cancer has not been elucidated. This study found that the expression of YTHDF2 was significantly upregulated in EOC tissues compared with normal ovarian tissues, indicating the involvement of YTHD F2 in promoting ovarian cancer. In addition, this study demonstrated that YTHDF2 promoted proliferation and migration, inhibited apoptosis, and reduced global mRNA m6A levels of EOC cell lines. Taken together, the results confirmed that YTHDF2 could promote proliferation and migration by decreasing global m6A levels.
MiR-145 is a newly discovered miR with significantly downregulated expression in breast cancer, cervical cancer, glioma, colon cancer, esophageal cancer, and nonsmall cell lung cancer [25, 26]. Many recent studies have found that microRNA is closely related to ovarian cancer $[27,28]$. Previous studies confirmed that miR-145 regulated different biological functions of ovarian cancer by targeting different target genes [17-20]. However, the expression pattern and the m6A-regulated role of miR145 in ovarian cancer were still unclear. The present study found that the expression levels of miR-145 and YTHDF2 had an inverse correlation in ovarian cancer tissues and cells. A crucial crosstalk occurred between miR-145 and YTHDF2 via a double-negative feedback loop, and YTHDF2 was the direct target gene of miR- 


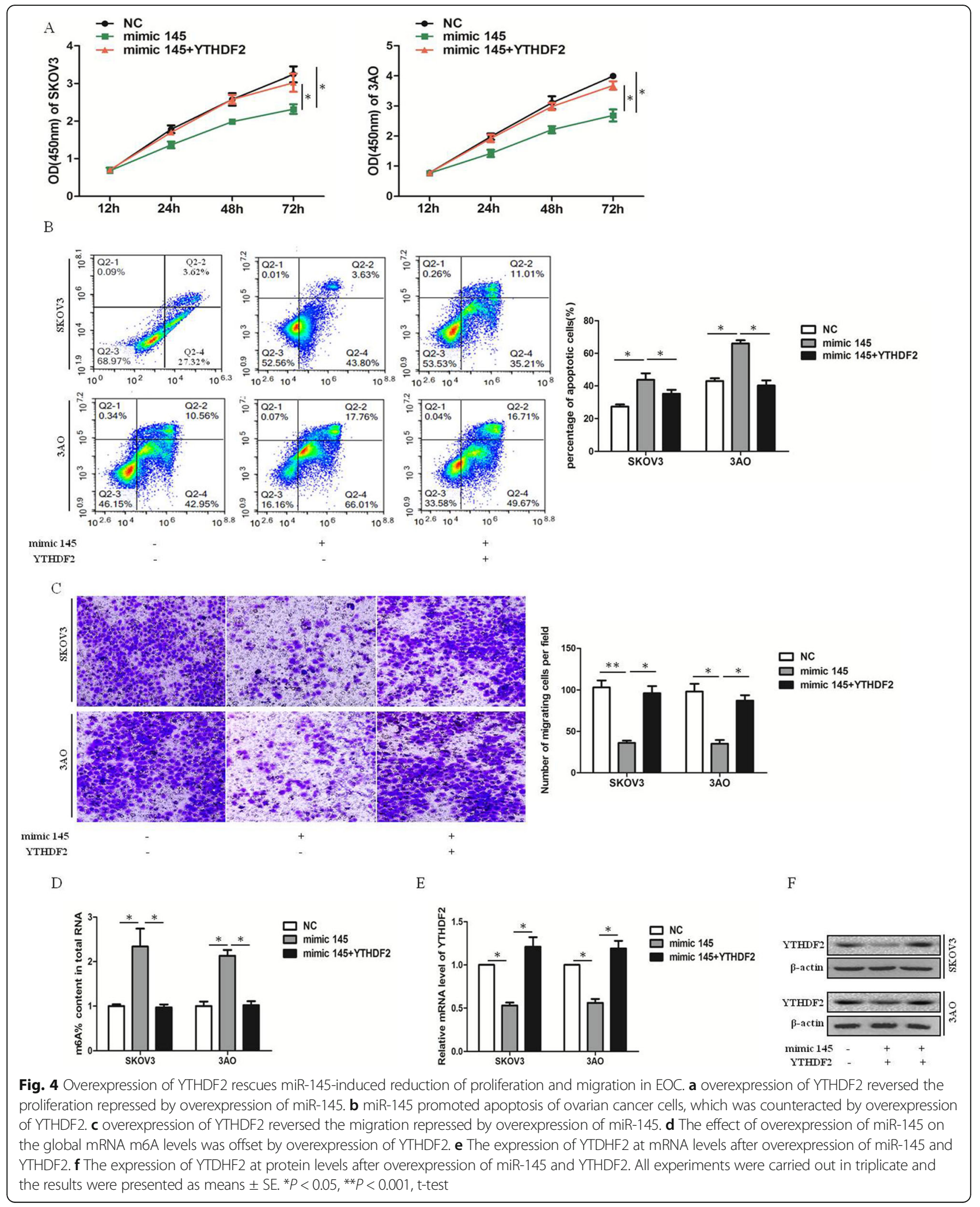

145. A correlation was found between the methylation modification of RNA m6A and miRNA. On the one hand, the miRNA-targeting site showed that m6A was enriched, and miRNA could positively regulate the activity of METTL3; on the other hand, the miRNA synthesis depended on m6A methylation modification [14, 15]. 
The findings confirmed that the primary microRNA was methylated by methylase METTL3 and recognized by the RNA-binding protein DGCR8. Drosha was recruited to cut the double-stranded RNA and produce the precursor miRNA (pre-miRNA) [14]. These results indicated that m6A was a marker for the posttranscriptional modification in miRNA biosynthesis. The present study demonstrated that YTHDF2 inhibited miR-145 expression. However, the molecular mechanism of YTHDF2 regulating miR-145 needs further exploration.

\section{Conclusions}

In summary, this study concluded that YTHDF2, an miR-145-repressed protein, promoted the proliferation and migration of ovarian cancer cells. It was novel in revealing the regulatory mechanism of YTHDF2 in ovarian cancer and demonstrating the involvement of miR-145 and YTHDF2 in m6A modification and progression of EOC. The findings on YTHDF2 and miR-145 may provide potential therapeutic targets of EOC.

\section{Acknowledgements}

Not applicable.

\section{Authors' contributions}

Jie Li conceived and designed the experiments. Lei Wu and Yun Zhang performed the experiments. Jie Li and Meili Pei analyzed the data. Jie Li, Lei Wu and Meili Pei wrote the paper. All authors read and approved the final manuscript.

\section{Funding}

This work was supported by National Natural Science Foundation of China (No.81702577), and Natural Science Basic Research Program of Shaanxi (No. 2018JQ8035), and the Fundamental Research Funds for the Central Universities (No. XZY012019105).

\section{Availability of data and materials}

The datasets during and/or analysed during the current study available from the corresponding author on reasonable request.

\section{Ethics approval and consent to participate}

Not applicable.

\section{Consent for publication}

Not applicable.

\section{Competing interests}

The authors declare that they have no competing interests.

\section{Author details}

'Department of Pathology, the First Affiliated Hospital of Xi'an Jiaotong University, 277 West Yanta Road, Xi'an 710061, Shaanxi, China. ${ }^{2}$ Department of Gynecology and Obstetrics, the First Affiliated Hospital of Xi'an Jiaotong University, Xi'an, China.

Received: 3 June 2020 Accepted: 14 September 2020 Published online: 18 September 2020

\section{References}

1. Stewart C, Ralyea C, Lockwood S. Ovarian Cancer: an integrated review. Semin Oncol Nurs. 2019;35(2):151-6.

2. Kujawa KA, Lisowska KM. Ovarian cancer--from biology to clinic. Postepy Hig i Med Doswiadczalnej (Online). 2015;69:1275-90.
3. Eisenhauer EA. Real-world evidence in the treatment of ovarian cancer. Annals Oncol. 2017;28(suppl_8):viii61-5.

4. Lan Q, Liu PY, Haase J, Bell JL, Huttelmaier S, Liu T. The critical role of RNA m (6) a methylation in Cancer. Cancer Res. 2019;79(7):1285-92.

5. Yu S, Li X, Liu S, Yang R, Liu X, Wu S. N (6)-Methyladenosine: a novel RNA imprint in human Cancer. Front Oncol. 2019;9:1407.

6. He L, Li H, Wu A, Peng Y, Shu G, Yin G. Functions of N6-methyladenosine and its role in cancer. Mol Cancer. 2019;18(1):176.

7. Zhang S, Zhao BS, Zhou A, Lin K, Zheng S, Lu Z, Chen Y, Sulman EP, Xie K, Bogler $\mathrm{O}$, et al. $\mathrm{m}(6)$ a Demethylase ALKBH5 maintains Tumorigenicity of Glioblastoma stem-like cells by sustaining FOXM1 expression and cell proliferation program. Cancer Cell. 2017;31(4):591-606 e596.

8. Ma JZ, Yang F, Zhou CC, Liu F, Yuan JH, Wang F, Wang TT, Xu QG, Zhou WP, Sun SH. METTL14 suppresses the metastatic potential of hepatocellular carcinoma by modulating N(6) -methyladenosinedependent primary MicroRNA processing. Hepatology (Baltimore). 2017; 65(2):529-43.

9. Vu LP, Pickering BF, Cheng Y, Zaccara S, Nguyen D, Minuesa G, Chou T, Chow A, Saletore $Y$, MacKay M, et al. The N(6)-methyladenosine (m(6)a)forming enzyme METTL3 controls myeloid differentiation of normal hematopoietic and leukemia cells. Nat Med. 2017;23(11):1369-76.

10. Muller S, Glass M, Singh AK, Haase J, Bley N, Fuchs T, Lederer M, Dahl A, Huang $\mathrm{H}$, Chen J, et al. IGF2BP1 promotes SRF-dependent transcription in cancer in a m6A- and miRNA-dependent manner. Nucleic Acids Res. 2019; 47(1):375-90.

11. Wang X, Lu Z, Gomez A, Hon GC, Yue Y, Han D, Fu Y, Parisien M, Dai Q, Jia $\mathrm{G}$, et al. N6-methyladenosine-dependent regulation of messenger RNA stability. Nature. 2014;505(7481):117-20.

12. Du H, Zhao Y, He J, Zhang Y, Xi H, Liu M, Ma J, Wu L. YTHDF2 destabilizes $\mathrm{m}(6)$ A-containing RNA through direct recruitment of the CCR4-NOT deadenylase complex. Nat Commun. 2016;7:12626.

13. Yang Z, Li J, Feng G, Gao S, Wang Y, Zhang S, Liu Y, Ye L, Li Y, Zhang $X$. MicroRNA-145 modulates N(6)-Methyladenosine levels by targeting the $3^{\prime}$-Untranslated mRNA region of the N(6)-Methyladenosine binding YTH domain family 2 protein. J Biol Chem. 2017;292(9):3614-23.

14. Alarcon CR, Lee H, Goodarzi H, Halberg N, Tavazoie SF. N6-methyladenosine marks primary microRNAs for processing. Nature. 2015;519(7544):482-5.

15. Alarcon CR, Goodarzi H, Lee H, Liu X, Tavazoie S, Tavazoie SF. HNRNPA2B1 is a mediator of $\mathrm{m}(6) \mathrm{A}$-dependent nuclear RNA processing events. Cell. 2015; 162(6):1299-308.

16. Zeinali T, Mansoori B, Mohammadi A, Baradaran B. Regulatory mechanisms of miR-145 expression and the importance of its function in cancer metastasis. Biomed Pharmacother. 2019;109:195-207.

17. Zhang S, Pei M, Li Z, Li H, Liu Y, Li J. Double-negative feedback interaction between DNA methyltransferase 3A and microRNA-145 in the Warburg effect of ovarian cancer cells. Cancer Sci. 2018;109(9):2734-45.

18. Li J, Lu J, Ye Z, Han X, Zheng X, Hou H, Chen W, Li X, Zhao L. 20(S)-Rg3 blocked epithelial-mesenchymal transition through DNMT3A/miR-145/ FSCN1 in ovarian cancer. Oncotarget. 2017:8(32):53375-86.

19. Li J, Zhang S, Zou Y, Wu L, Pei M, Jiang Y. miR-145 promotes miR-133b expression through c-myc and DNMT3A-mediated methylation in ovarian cancer cells. J Cell Physiol. 2020;235(5):4291-301.

20. Li J, Li X, Wu L, Pei M, Li H, Jiang Y. miR-145 inhibits glutamine metabolism through c-myc/GLS1 pathways in ovarian cancer cells. Cell Biol Int. 2019; 43(8):921-30.

21. Jia G, Fu Y, Zhao X, Dai Q, Zheng G, Yang Y, Yi C, Lindahl T, Pan T, Yang YG, et al. N6-methyladenosine in nuclear RNA is a major substrate of the obesity-associated FTO. Nat Chem Biol. 2011;7(12):885-7.

22. Li J, Meng S, Xu M, Wang S, He L, Xu X, Wang X, Xie L. Downregulation of $\mathrm{N}(6)$-methyladenosine binding YTHDF2 protein mediated by miR-493-3p suppresses prostate cancer by elevating N(6)-methyladenosine levels. Oncotarget. 2018;9(3):3752-64.

23. Zhong L, Liao D, Zhang M, Zeng C, Li X, Zhang R, Ma H, Kang T. YTHDF2 suppresses cell proliferation and growth via destabilizing the EGFR mRNA in hepatocellular carcinoma. Cancer Lett. 2019:442:252-61.

24. Hou J, Zhang H, Liu J, Zhao Z, Wang J, Lu Z, Hu B, Zhou J, Feng M, Shen B, et al. YTHDF2 reduction fuels inflammation and vascular abnormalization in hepatocellular carcinoma. Mol Cancer. 2019;18(1):163.

25. Xu WX, Liu Z, Deng F, Wang DD, Li XW, Tian T, Zhang J, Tang JH. MiR-145: a potential biomarker of cancer migration and invasion. Am J Transl Res. 2019;11(11):6739-53 
26. Xu L, Zhang Y, Tang J, Wang P, Li L, Yan X, Zheng X, Ren S, Zhang M, Xu M. The prognostic value and regulatory mechanisms of microRNA-145 in various tumors: a systematic review and meta-analysis of 50 studies. Cancer Epidemiol Biomark Prev. 2019;28(5):867-81.

27. Zhang W, Wang $Q$, Yu M, Wu N, Wang H. MicroRNA-145 function as a cell growth repressor by directly targeting c-Myc in human ovarian cancer. Technol Cancer Res Treat. 2014;13(2):161-8.

28. Ghafouri-Fard S, Shoorei H, Taheri M. miRNA profile in ovarian cancer. Exp Mol Pathol. 2020;113:104381.

\section{Publisher's Note}

Springer Nature remains neutral with regard to jurisdictional claims in published maps and institutional affiliations.

Ready to submit your research? Choose BMC and benefit from:

- fast, convenient online submission

- thorough peer review by experienced researchers in your field

- rapid publication on acceptance

- support for research data, including large and complex data types

- gold Open Access which fosters wider collaboration and increased citations

- maximum visibility for your research: over $100 \mathrm{M}$ website views per year

At $\mathrm{BMC}$, research is always in progress.

Learn more biomedcentral.com/submissions 